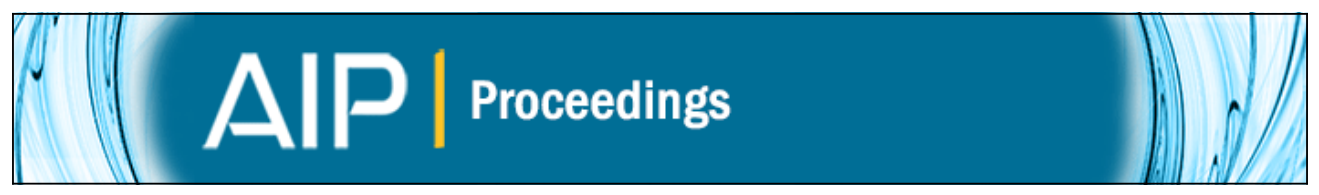

Detecting Dark Matter in the MSSM with Non-Universal Higgs Masses

Pearl Sandick

Citation: AIP Conference Proceedings 1200, 1027 (2010); doi: 10.1063/1.3327529

View online: http://dx.doi.org/10.1063/1.3327529

View Table of Contents:

http://scitation.aip.org/content/aip/proceeding/aipcp/1200?ver=pdfcov

Published by the AIP Publishing

Articles you may be interested in

Neutralino Dark Matter in MSSM Models with Non-Universal Higgs Masses

AIP Conf. Proc. 1241, 450 (2010); 10.1063/1.3462670

Thermal right-handed sneutrino dark matter in the Next-to-MSSM

AIP Conf. Proc. 1200, 1071 (2010); 10.1063/1.3327541

WIMPless Dark Matter

AIP Conf. Proc. 1200, 1059 (2010); 10.1063/1.3327538

Dark matter signals in deflected mirage mediation

AIP Conf. Proc. 1200, 1019 (2010); 10.1063/1.3327527

MSSM Dark Matter Without Prejudice

AIP Conf. Proc. 1200, 1015 (2010); 10.1063/1.3327526 


\title{
Detecting Dark Matter in the MSSM with Non-Universal Higgs Masses
}

\author{
Pearl Sandick \\ Theory Group and Texas Cosmology Center, \\ The University of Texas at Austin, TX 78712, USA
}

\begin{abstract}
We discuss the direct detection prospects for neutralino dark matter via elastic scattering in variations of the MSSM with non-universal supersymmetry-breaking contributions to the Higgs masses. Taking as our starting point the CMSSM, in which supersymmetry-breaking contributions to all scalar masses are universal, we examine scenarios in which both Higgs scalar masses are non-universal by the same amount (NUHM1) and scenarios in which the Higgs scalar masses are independently non-universal (NUHM2).
\end{abstract}

Keywords: Supersymmetry, Dark Matter

PACS: $11.30 . \mathrm{Pb}, 95.35 .+\mathrm{d}$

The Constrained Minimal Supersymmetric Extension of the Standard Model (CMSSM), in which the soft supersymmetry-breaking contributions to the squark, slepton and Higgs scalar masses are universal at some GUT input scale, is often studied and its phenomenology fairly well understood. However, while it may be the simplest scenario, it is by no means the most plausible version of the MSSM. Here, we present the results of a recent study on the direct detection prospects in models with non-universal supersymmetry-breaking contributions to the Higgs masses [1].

The CMSSM is parametrized by four continuous parameters specified at the SUSY GUT scale and a sign choice: the universal gaugino mass, $m_{1 / 2}$, the universal scalar mass, $m_{0}$, the universal value for the trilinear couplings, $A_{0}$, the ratio of the Higgs vevs, $\tan (\beta)$, and the sign of the Higgs mixing parameter, $\mu$. In this scenario, the effective Higgs masses are $m_{1}=m_{2}=m_{0}$ at the GUT scale, and the electroweak vacuum conditions fix $|\mu|$ and the pseudoscalar Higgs mass, $m_{A}$. In the NUHM1, in which the effective Higgs masses are assumed to be universal at the GUT scale (though that value may differ from $m_{0}$ ) there is one additional input, which may be taken as $\mu, m_{A}$, or the GUT-scale value of the Higgs masses $m_{1}=m_{2}$. Similarly, the NUHM2, in which $m_{1}$, $m_{2}$, and $m_{0}$ are all independent, may be parametrized by additional inputs $\mu$ and $m_{A}$, or by both GUT-scale effective Higgs masses. In all cases, we assume that the lightest neutralino is the dark matter candidate.

In each scenario, the Renormalization Group Equations of the MSSM are used to determine the low-scale observables, and constraints from colliders and cosmology are applied. Here we calculate the direct detection cross sections for models which are phenomenologically and cosmologically viable, i.e. models not excluded by constraints from colliders, and where the upper limit on the relic density of neutralinos is respected. Direct detection experiments take the measured dark matter abundance, $\Omega_{C D M}$, as a prior. Therefore, if the calculated neutralino abundance $\Omega_{\chi}<\Omega_{C D M}$ [2], we assume CP1200, $17^{\text {th }}$ International Conference on Supersymmetry and the Unification of Fundamental Interactions,
edited by P. Nath, G. Alverson, and B. Nelson

(C) 2009 American Institute of Physics 978-0-7354-0737-4/09/\$25.00 

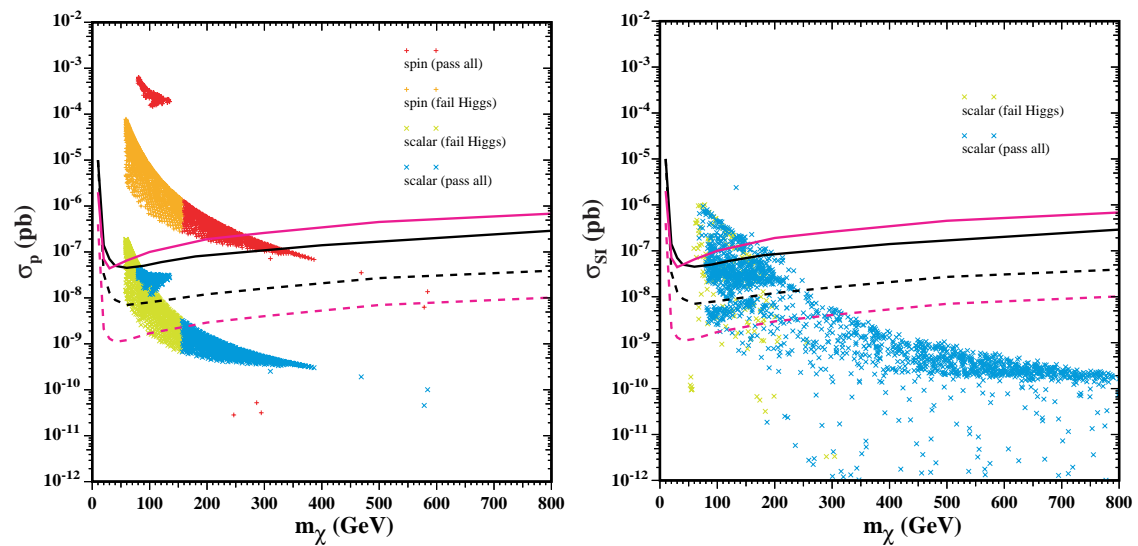

FIGURE 1. In the left plane, we show cosmologically viable neutralino-nucleon elastic scattering cross sections as functions of neutralino mass for $\tan \beta=10$, and $A_{0}=0$. The right panel shows the entire potential range of neutralino-nucleon cross sections as functions of neutralino mass for the CMSSM, with $5 \leq \tan \beta \leq 55,100 \mathrm{GeV} \leq m_{1 / 2} \leq 2000 \mathrm{GeV}, 0 \leq m_{0} \leq 2000 \mathrm{GeV}$, and $-3 m_{1 / 2} \leq A_{0} \leq 3 m_{1 / 2}$ We consider $\mu<0$ only for $\tan \beta<30$. Also shown are upper limits on the SI dark matter scattering cross section as specified in the text. For further details on this and other figures, please refer to [1].

a secondary source of cold dark matter, and rescale the plotted neutralino-nucleon cross sections by a factor $\Omega_{\chi} / \Omega_{C D M}$, so that comparison with direct searches is possible.

In Fig. 1, we present the neutralino-nucleon elastic scattering cross section as a function of neutralino mass in the CMSSM. In the left panel, only one slice of the parameter space is exhibited. We have separated out the cross section for spin-dependent (SD) scattering (red/orange) from the scalar or spin-independent (SI) cross section (blue/green). Here and throughout, we assume the current limits on the $\mathrm{BR}(B \rightarrow \mu \mu)$ and the $\mathrm{BR}(b \rightarrow s \gamma)$ are respected, as well as the lower limit on the chargino mass, and differentiate regions in which the scalar Higgs mass is below the LEP limit (green/orange) and above the LEP limit (blue/red). We also plot the limits on the SI cross section from CDMS II [3] (solid black line) and XENON10 [4] (solid pink line), as well as the sensitivities projected for XENON100 [5] (or a similar 100-kg liquid noble-gas detector such as LUX, dashed pink line) and SuperCDMS at the Soudan Mine [6] (dashed black line). One can see that the SD cross sections are generally larger, though current upper limits are larger than $10^{-2} \mathrm{pb}$.

Both the SI and SD cross sections in the left panel contain two distinct regions, that arising from the focus-point region at $m_{\chi} \lesssim 150 \mathrm{GeV}$ and relatively large $\sigma$, and that from the coannihilation strip. In the coannihilation strip, $50 \mathrm{GeV}<m_{\chi}<400 \mathrm{GeV}$, where the lower limit on $m_{\chi}$ is a result of the LEP constraint on the chargino mass, and the upper limit on $m_{\chi}$ corresponds to the end-point of the coannihilation strip for $\tan \beta=10$. At larger values of $\tan \beta$, we also find a rapid annihilation funnel in the CMSSM parameter space.

In the right panel of Fig. 1, we scan over all CMSSM parameters. One can see that there is an upper limit on the SI cross section as a function of neutralino mass, and also 

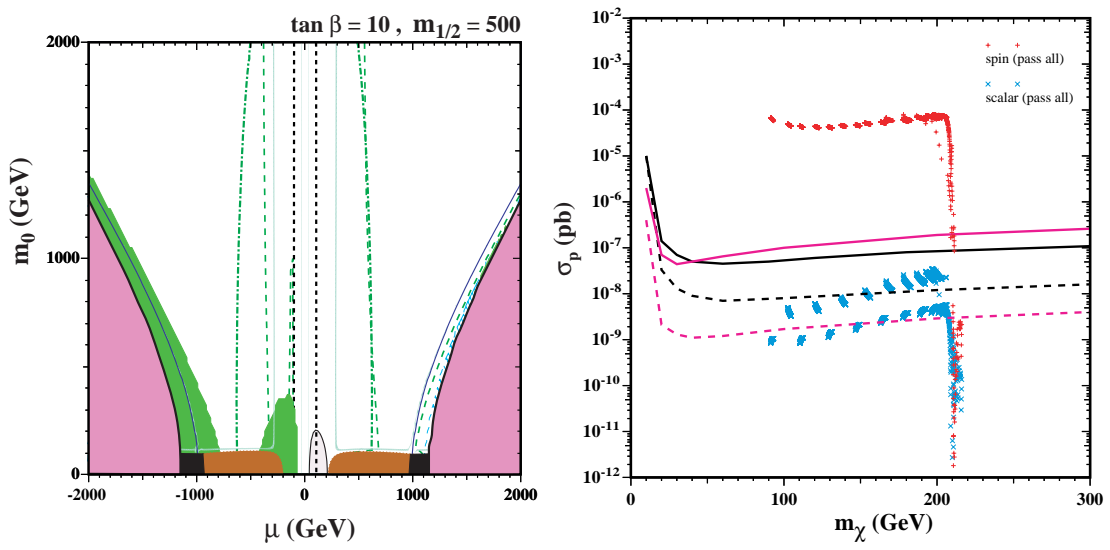

FIGURE 2. The left panel shows an NUHM1 $\left(\mu, m_{0}\right)$ plane for $m_{1 / 2}=500 \mathrm{GeV}, \tan \beta=10$, and $A_{0}=0$. The right panel shows the corresponding neutralino-nucleon elastic scattering cross sections for cosmologically-viable models.

that at low $m_{\chi}<300 \mathrm{GeV}$, cross sections generally exceed $10^{-9} \mathrm{pb}$. The largest cross sections, already excluded by CDMS and XENON10, come primarily from the focus point region at large $\tan \beta$.

In the NUHM1, the additional parameter results in additional regions where all constraints are satisfied, selectron coahhihilation strips and crossover regions. In the left panel of Fig. 2, we show an example slice of NUHM1 parameter space, a $\left(\mu, m_{0}\right)$ plane. The cosmologically-preferred (turquoise) shaded strips, from large $|\mu|$ in, are: rapid annihilation funnels rising up at $|\mu| \approx 1000 \mathrm{GeV}$ and arcing outwards (note that for $\mu<0$ the entire funnel is excluded by the $\operatorname{BR}(b \rightarrow s \gamma)$ ), selectron coannihilation strips bordering the black excluded regions at low $m_{0}$, between $|\mu| \approx 1000 \mathrm{GeV}$ and $|\mu| \approx 300$ $\mathrm{GeV}$ at low $m_{0}$ are the familiar stau coannihilation strips, and there are vertical crossover strips at $|\mu| \approx 300 \mathrm{GeV}$, where the LSP is in a mixed bino-Higgsino state. The resulting $\chi$-nucleon cross sections are shown in the right panel of Fig. 2. Most cross sections are clustered at $m_{\chi} \sim 215 \mathrm{GeV}$ due to the fact that the lightest neutralino is bino-like over most of the plane. The smallest cross sections shown come from the funnel or other regions where the relic density is suppressed (and therefore the cross section is scaled). The largest cross sections come from the crossover strip, near its intersection with the coannihilation strip, and for $\mu>0$. Between the crossover strips, moving to smaller $|\mu|$, $m_{\chi}$ decreases, as does the relic density of neutralinos, leading to strips in the right panel of scaled cross sections trailing down to $m_{\chi} \approx 100 \mathrm{GeV}$.

Finally, in Fig. 3 we show the entire potential ranges for the SI $\chi$-nucleon elastic scattering cross section as a function of neutralino mass in both the NUHM1 and NUHM2. As a result of the additional freedom in the Higgs sector, and since the SI elastic scattering cross sections receive important contributions from Higgs exchange, there is more variability in the cross sections in the NUHM1 and NUHM2 than in the CMSSM. Differences arise primarily from additional regions of parameter space where the lightest neutralino is in a mixed bino-Higgsino state, such as the crossover region. 

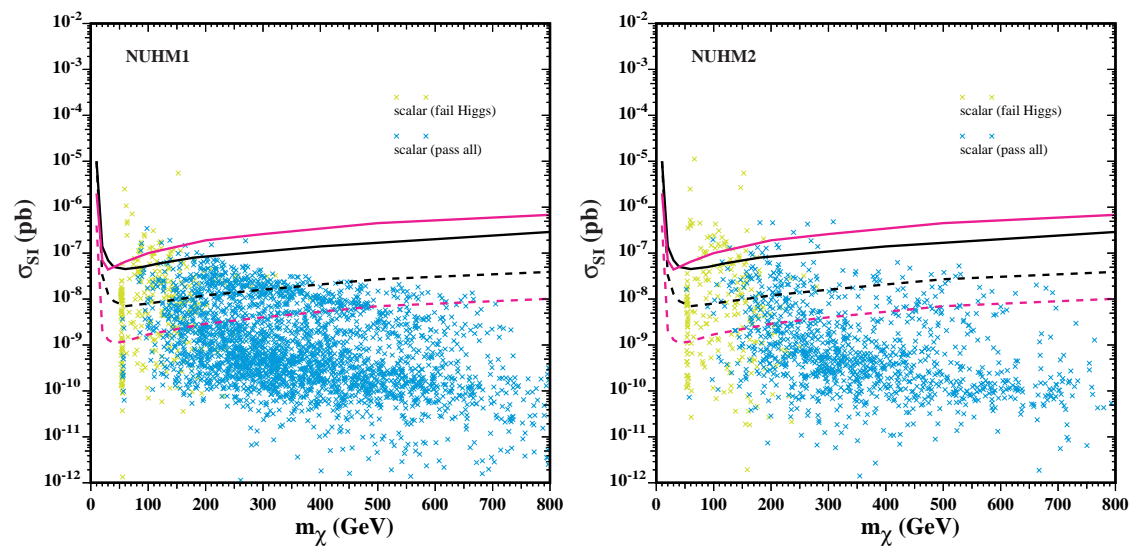

FIGURE 3. We show the entire potential ranges in the NUHM1 and NUHM 2 of the SI neutralinonucleon cross sections with respect to neutralino mass. In both plots, we scan $5 \leq \tan \beta \leq 55,100 \mathrm{GeV}$ $\leq m_{1 / 2} \leq 2000 \mathrm{GeV}, 0 \leq m_{0} \leq 2000 \mathrm{GeV}$, and $-3 m_{1 / 2} \leq A_{0} \leq 3 m_{1 / 2}$. The GUT-scale values of $m_{1}$ and $m_{2}$ are in the range $(-2000,2000) \mathrm{GeV}$, with $m_{1}=m_{2}$ in the NUHM1.

In both the NUHM1 and NUHM2, there may be significantly larger cross sections at larger $m_{\chi}$ than would be expected in the CMSSM. These cross sections may be probed by the next generation of direct detection experiments for $m_{\chi}$ as large as $\sim 700 \mathrm{GeV}$, while in the CMSSM there is no hope for detection with these instruments for $m_{\chi} \gtrsim 350$ $\mathrm{GeV}$. In addition, there are viable cross sections at very low $m_{\chi}$ with rather low cross sections. If nature has given us a neutralino of this character, then we may not directly detect dark matter for some time, but the LHC may find supersymmetric particles fairly quickly. In either scenario, we will know that the CMSSM is not an adequate description of nature. No single experiment can tell us everything we would like to know about how supersymmetry is broken, but the next generation of direct detection experiments may give us the first hint.

\section{ACKNOWLEDGMENTS}

This material is based upon work supported by the National Science Foundation under Grant No. PHY-0455649.

\section{REFERENCES}

1. J. Ellis, K. A. Olive and P. Sandick, arXiv:0905.0107 [hep-ph].

2. E. Komatsu et al. [WMAP Collaboration], Astrophys. J. Suppl. 180, 330 (2009).

3. Z. Ahmed et al. [CDMS Collaboration], Phys. Rev. Lett. 102, 011301 (2009).

4. J. Angle et al. [XENON Collaboration], Phys. Rev. Lett. 100, 021303 (2008).

5. E. Aprile, L. Baudis and f. t. X. Collaboration, arXiv:0902.4253 [astro-ph.IM].

6. SuperCDMS Development Project, Fermilab Proposal 0947, October 2004. 